\title{
The value of serum ferritin and pre-albumin in distinguishing primary mild COVID-19 patients and the patients with recovery positive virus detection after discharge
}

\author{
liwen luo ${ }^{1}$, Xiuying $\operatorname{Jian}^{2}$, and Zhiqiang $\operatorname{Tian}^{3}$ \\ ${ }^{1}$ Affiliation not available \\ ${ }^{2}$ Chongqing Medical University Affiliated Second Hospital \\ ${ }^{3}$ Army Military Medical University
}

May 14, 2020

\begin{abstract}
Objectives: To distinguish between primary mild COVID-19 patients and the patients with recovery positive virus detection after discharge (recovery positive patients), the clinical characteristics and biochemical indicators were analyzed. Methods: By evaluation of correlations between biochemical indicators and serum ferritin (FE) through univariate and multivariate analyses, we evaluated the differences of FE index and analyzed the effectiveness of the FE* pre-albumin (PA) on discrimination between primary mild and recovery positive COVID-19 patients. The area under curve (AUC) and a predictive nomogram were further employed to evaluate the effectiveness of discrimination. Results: In comparison with recovery positive patients, liver damagerelated indicators such as serum FE and PA were significantly lower in primary COVID-19 patients with mild symptoms. Area under the ROC curve values of $\mathrm{FE}, \mathrm{PA}$ and $\mathrm{FE} * \mathrm{PA}$ were $0.81,0.78$ and 0.83 , respectively. In combination with multivariate analysis and nomogram, $\mathrm{FE}$ and $\mathrm{FE}^{*} \mathrm{PA}$ can be considered as reasonable evaluation systems for predicting primary mild and recovery positive patients. Conclusions: FE*PA was an independent predictive biomarker for distinguishing primary mild and recovery positive COVID-19 cases.
\end{abstract}

\section{Introduction}

The new coronavirus pneumonia is a serious respiratory disease, and its causative agent is Severe Acute Respiratory Syndrome Coronavirus 2 (SARS-CoV-2), a betacoronavirus ${ }^{[1]}$. Currently, this disease has been formally termed COVID-19 by the World Health Organization (WHO) ${ }^{[2]}$. Due to its rapid onset, strong infectivity, general susceptibility to the population, and rapid changes in disease conditions ${ }^{[3,4]}$, it has been classified as a serious infectious disease all over the world. Previous studies have confirmed that COVID-19 can be transmitted from person to person, mainly through droplets, contact, and possibly through feces and aerosols. After infection with SARS-CoV-2 virus, more than $80 \%$ patients appear to be with mild symptom $^{[5]}$, however, while these mild patients have the risk to get worse, they have considerable ability to spread the disease, suggesting necessary protective measure for these mild cases.

Recently there are some clinical reports in China that some recovery patients' RT-PCR tests for COVID-19 are positive again during the 2 -week home isolation after discharge from hospital. However, these recovery positive patients have not been observed the ability to spread the infection once more. Therefore, during the pandemic period, different diagnosis and treatment schemes should be adopted for the primary mild patients and the recovery positive patients by distinguishing the types of diseases, thereby reducing the social and medical burden to combat with this disease. Although the best and most reliable test to distinguish them with other groups and to assess their infectivity is the antibody test, specific antibodies against COVID-19 already exist in the serum of both primary and recovery positive patients, so serology can prove whether or 
not COVID-19 has been infected, but it cannot help us effectively distinguish the primary patients from the recovery positive patients. Therefore, biochemical indicators may be more helpful to distinguish primary mild COVID-19 and the patients with recovery positive virus detection.

Studies on patients with severe symptoms showed that COVID-19 induced a large number of inflammatory factors and also led to damage to the liver and kidney ${ }^{[6]}$. The conditions were worse and the mortality rate was higher in elder patients in severe situation ${ }^{[7]}$. However, organ damage caused by COVID-19 in primary mild and recovery positive patients still remains unclear. In our work, we analyzed and compared biochemical indicators and internal organs damage caused by COVID-19 between the primary mild and recovery positive patients. Through the evaluation of liver injury related serological indicators, we investigated whether liver damage in patients with mild symptoms was more severe than those in recovery positive cases.

Serum ferritin (FE) and pre-albumin (PA) serological markers closely related to liver function ${ }^{[8]}$. When liver damage occurs, the levels of FE and PA significantly decrease. In the cases of liver fibrosis or liver cancer, due to the dysregulation of synthetic function in the liver, the levels of synthesis related serum protein, such as serum ferritin, pre-albumin, and hemoglobin decrease, and the coagulation weakens ${ }^{[9,10]}$. Therefore, these significantly changed serological indicators can be used as predictive biomarkers to distinguish the types of patients and judge the disease severity. Considering that FE and PA were biomarkers of liver damage, we employed $\mathrm{FE} * \mathrm{PA}$ as an indicator to distinguish between primary mild and recovery positive COVID-19 patients.

In this work, through a comparative analysis of the serological indicators of two types COVID-19 patients, we found that the degree of liver damage in primary mild COVID-19 patients was greater than that in recovery positive patients, and evaluation of $\mathrm{FE}$ and PA levels was effective to discriminate between primary mild and recovery positive cases. Our study will provide guidance for doctors to develop effective treatment plans specifically for patients in different infection status and prevent the COVID-19 transmission.

\section{Research Design and Methods}

\section{Research Design}

A total of 184 patients including 149 primary mild and 35 recovery positive COVID-19 cases were admitted to the Central Hospital of Xiaogan City between January 2020 and March 2020. The study was a retrospective study of hospitalized patients with a definitive diagnosis of mild and recurrent positive COVID-19 patients by the diagnostic criteria. The main basis for the diagnosis of primary mild patients was positive viral nucleic acid test accompanying with or without multiple patchy shadows in both lungs in the CT test,fever, mild digestive tract symptoms (nausea, vomiting, diarrhea, abdominal pain and anorexia), and mild respiratory symptoms (cough, sputum, difficulty breathing, chest tightness and throat discomfort). After the primary patients' nucleic acid test twice were negative at intervals of 2 days during treatment, the CT results showed no lung infection and the clinical symptoms were significantly improved or disappeared, these patients were thought recovered from the COVID-19 infection and were isolated at home after discharge from hospital. All discharged patients need to undergo nucleic acid testing every five day for at least two weeks. The patients were classified as recovery positive patients if there are positive RT-PCR test results during the 2-week isolation period at home, no matter whether the patients were accompanied by clinical symptoms. In addition, patients did not consume any drugs affecting levels of FE and PA and liver function during home quarantine.

For the primary or recovery positive patients, the biochemical parameters used for statistical analysis in this study were collected from the initial biochemical detection after admission. The medical history records of patients before admission were reviewed to exclude cases failed to meet the criteria. In order to reduce the impact of primary disease on biochemical indicators, the following strict exclusion criteria were applied based on the medical history: previous suffering from primary pancreatic benign and malignant tumors; primary hepatitis, liver benign and malignant tumors; primary cholecystolithiasis, cholecystitis and gallbladder cancer; primary nephritis, renal failure, kidney benign and malignant tumors; primary autoimmune diseases such as SLE, HIV, etc. The study was conducted in accordance with the Declaration of Helsinki of the 
World Medical Association and approved by the ethics committee of the Army Military Medical University. All patients signed informed consent forms.

\section{Data Collection, Definitions, and Quantitative Determination of FEPA}

To standardize the blood test results, the data of the first routine blood examination and biochemical test were carried out within two days of admission. Laboratory test results, clinical features, epidemiological and pathological test results were obtained from the official website of the Central Hospital of Xiaogan and analyzed independently by two physicians. Blood FE*PA was calculated by multiplying the FE and the PA. The biological parameters of all the data for statistical analysis in this study were in consistence with the experimental data of the hospital. To make a more reasonable predictive nomogram, FEPA was defined as FE*PA divided by 1000 .

\section{Statistical Analysis}

Data analysis was performed by SPSS 22.0 and GraphPad Prism 6.0 software packages. Continuous variables with normal distributions were shown as mean \pm standard deviation (SD). Counting data was analyzed by the $\chi^{2}$ test and multivariate analysis was performed using multivariate logistics analysis. Odds ratios (ORs) and $95 \%$ confidence intervals (CIs) were determined for variables of interest. Spearman R correlations were used to analyze correlations between FE and other serum indictors. ROC curves were used to analyze the efficacy of FE, PA and FEPA for diagnosis of pancreatitis types. $\mathrm{P}$ values $<0.05$ were considered as statistically significant.

\section{Results}

\section{Clinical Characteristics}

A total of 184 patients were investigated in this study, including 149 mild COVID-19 patients and 35 recovery positive patients. The clinical characteristics of mild COVID-19 patients and recovery positive patients were shown in Table $\mathbf{1}$. The probability of primary COVID-19 patients accompanied by the gastrointestinal symptoms, respiratory symptoms and fever are $38.93 \%, 79.19 \%$, and $82.55 \%$, respectively. The probability of recovery positive COVID-19 patients accompanied by the gastrointestinal symptoms, respiratory symptoms and fever are $20 \%, 48.57 \%$, and $22.86 \%$, respectively. The probability of occurrence of gastrointestinal symptoms, respiratory symptoms and fever was significantly different between the primary and recovery positive patients $(\mathrm{P}<0.05)$. The detection data of biochemical indicators were shown in Table 2 Excluding significantly increased in Fibrinogen, APTT and TT, serological indicators in primary mild COVID-19 patients for RBC, HGB, EO, TCa, FE, TP, ALB, PA, CRE and UA are significantly decreased compared to the serological indicators in recovery positive patients. Moreover, FE*PA was significantly higher in recovery positive patients. In addition, a differential analysis of serum levels of APTT, HGB, ALB, FE, PA and FE*PA related with liver damage for the two types of COVID-19 disease was shown in Figure 1 .

\section{Relationships between FE level and other biomarkers}

The correlations between various serum indicators and FE level were analyzed by Pearson's correlations (Table 3 ). Among the primary mild and recovery positive COVID-19 patients, the FE level positively correlated with the levels of serum indicators of liver injury including RBC, HGB, TCa, TP, ALB, PA and TBIL, which weaken the synthesis function. However, FE negatively correlated with the levels of coagulation function indicators such as PT, APTT and fibrinogen. Moreover, PA was the highest correlated factor with $\mathrm{FE}$ with $\mathrm{R}$ value at 0.5 .

\section{Multivariate analysis and nomogram prediction}

Multiple logistic regression analysis was applied to evaluate the differences between mild and recovered COVID-19 patients. Statistically significant variables were shown in the Table 4 . PT[OR 0.55, (95\% CI, 0.36-0.85), P 0.007], FE[OR 1.22, (95\% CI, 1.12-1.32), P 0.000], ALT[OR 0.97, (95\% CI, 0.94-1.00), P 0.026], TBIL[OR 0.79, (95\% CI, 0.94-1.00), P 0.031], LYMPH[OR 0.32, (95\% CI, 0.12-0.84), P 0.020], DBIL[OR 
1.76, (95\% CI, 1.15-2.69), P 0.009] and $\mathrm{FE}^{*} \mathrm{PA}[\mathrm{OR}$ 1.00, (95\% CI, 1.00-1.01), P 0.000] were found to be independent predictors in distinguishing the two subtypes of patients. Furthermore, the nomogram was developed to predict the probability of primary mild and recovery positive COVID-19 patients (Fig. 2) - Through comprehensive statistical analysis of various parameters in multivariate analysis, FE, LYMPH, DBIL and FEPA indicators have been demonstrated to be effectively distinguish and predict primary and recovery positive cases.

\section{The validation of nomogram performances}

Although multiple differences between primary mild and recovery positive COVID-19 patients were identified by univariate analysis of clinical characteristics, $\mathrm{FE}^{*} \mathrm{PA}$ values exhibited great potential to be the most powerful indicator to distinguish between two kinds of cases according to the statistic analysis. To valid the developed nomogram, values of FE, PA and FE*PA in both COVID-19 groups were analyzed. The AUC value for distinguishing between two types of patients by FE*PA was 0.83 , and the selecting optimal sensitivity and specificity were 0.77 and 0.58 , respectively. The AUC values of FE and PA were 0.81 and 0.77 respectively, which were lower than that of FE*PA. Similarly, the sensitivity and specificity of FE and PA were lower than those of FE*PA (Figure 3 ). Our results showed that FEPA was an efficient indicator for distinguishing primary mild COVID-19 patients from recovery positive patients.

\section{Discussion}

The COVID-19 patients commonly presented with fever, gastrointestinal and respiratory symptoms, despite the incidence of these symptoms reflected significant differences between primary and recurrent patients, clinical characteristics are not reliable to distinguish and predict primary mild and recovery positive COVID19 patients precisely. In severe cases, COVID-19 can lead to obvious respiratory distress and/or hypoxemia, ARDS, septic shock and even death ${ }^{[11]}$. Previous studies reported that COVID-19 could cause the higher incidence of liver injury and dysfunction of multiple organs due to the excessive inflammatory reactions in severe patients rather than mild COVID-19 patients. This was indicated by abnormal ALT/AST levels as well as slightly increased bilirubin levels ${ }^{[6,12]}$. Recent studies confirmed that besides infecting the lungs and causing liver damage, COVID-19 can also induce blood vessels and kidney damage by binding the angiotensin converting enzyme $2(\mathrm{ACE} 2)^{[13,14]}$. Studies conducted in hospitalized COVID-19 patients demonstrated that nearly $40 \%$ patients were presented with proteinuria and hematuria. Moreover, a CT scan of the kidney showed a reduced density suggesting the kidney experienced inflammatory responses and edema. Up to date, there is no study of comparison of differences in liver and kidney injury between primary mild and recurrent COVID-19 patients. In the present study, we found that the indicators associated with liver damage, such as RBC, HGB, EO, TCa, FE, TP, ALB, PA, were significantly lower in the primary infected cases compared with recovery positive patients. Whereas, the biochemistries PT and APTT in primary COVID-19 patients elevated. In terms of renal function indicators, serum CRE and UA levels were significantly higher in primary cases, indicating that primary patients suffered from more severe kidney injury, and the specific antibodies against COVID-19 in recovered positive patients serum played a crucial role in alleviating the organ damage.

Although the recovered patients were tested with the positive virus detection, the inflammatory response and vascular injury effects caused by the COVID-19 were greatly reduced leading to the production of protective antibodies and significant reduction of the damage to patients. FE is involved in the storage and digestion of iron ${ }^{[15]}$. The level of $\mathrm{FE}$ is not only related to the hemoglobin production, but also a biomarker of liver-specific damage ${ }^{[16]}$. Spearman R correlations were performed to analyze the correlation between FE levels and other biochemistries. In summary, our results indicated that in patients with mild COVID-19 symptoms, the function of synthetic serum FE and PA weakened after liver damage, and the two indicators were correlated to each other.

According to the current data research, recovery positive patients may not have the ability to spread the disease to others, but primary mild COVID-19 patients, including some asymptomatic patients, have a stronger ability to spread the disease. Moreover, compared with recovery positive patients, the symptoms of the primary mild patients were significantly worse, and the prognosis may be worse. Therefore, during 
the COVID-19 outbreak, development and management of specific treatment strategies to different types of patients were important to limit the virus spreading. For primary COVID-19 patients, treatments need to be applied to reduce the potential risks for severe disease. For recovery positive patients, self-isolate at home was recommended, which could reduce the pressure on hospitals and society. Moreover, discrimination between primary and recovery positive cases was also important to clarify the ability and intensity of disease spreading among primary and recovery positive patients, and provide guidance for epidemiological prevention. Therefore, clinical symptoms or serological indicators were initially selected to distinguish between primary and recovery positive COVID-19 patients. However, discrimination between primary mild and recurrent positive patients based on clinical manifestations is not reliable since both types of cases show similar mild symptoms. To predict and distinguish between the primary and recovery positive COVID-19 cases, the differences of the serological indicators in patients were analyzed in this study. This will provide helpful information to the clinical diagnosis and development of suitable treatment plans during the epidemic outbreak. Multivariate analysis was employed to seek serum indicators related to discrimination. The significant parameters in the multivariable analysis were further selected for nomogram analysis, FE, LYMPH, DBIL and FEPA were proved to be capable of effectively predicting and distinguishing different types of patients indicating nomogram was reliable and easily accessible. Combining FE and PA (FE*PA) showed the highest correlation values implying the potential to be an indicator to distinguish between primary mild and recurrent patients. Furthermore, the analysis of AUC value, sensitivity and specificity demonstrated FE*PA was the optimal predictor compared with PA and FE, and the developed nomogram exhibited an acceptable performance for patient discrimination and prediction

In conclusion, our study suggested that liver and kidney damage in COVID-19 patients with mild symptoms was significantly more severe than that in recovery positive patients. Moreover, FE*PA has potential to be an independent predictive biomarker for distinguishing COVID-19 patients. This may provide an alternative method to rapidly diagnose mild and recovered COVID-19 patients without nucleic acid tests, CT tests, and medical history and improve treatment efficiency.

\section{Acknowledgements}

We thank all patients involved in the study.

\section{Author Contributions}

Liwen Luo, Xiuying Jian collected and interpreted the data. Liwen Luo analyzed the data and wrote the manuscript. Zhiqiang Tian revised the the manuscript. All authors read and approved the final manuscript.Notes: Liwen Luo and Xiuying Jian contributed equally to this work and should be considered as co-first authors. Conflicts of Interest : The authors declare no conflict of interest.

\section{Funding}

This work was supported by the National Key Research and Development Project (Grant/Award Numbers: 2016YFA0502200, 2016YFA0502203 and 2017YFC1308600). The Research Program of Foundation Science and Application Technology of Chongqing (Grant/Award Numbers: cstc2018jcyjA1826 and cstc2015jcyjA10105). Basic Medical College Foundation of Army Military Medical University (2019JCZX10).

\section{References}

1. Chen Y, Liu Q, Guo D:Emerging coronaviruses: Genome structure, replication, and pathogenesis . Journal of medical virology 2020,92 (4):418-423.

2. Carlos WG, Dela Cruz CS, Cao B, Pasnick S, Jamil S: Novel Wuhan (2019-nCoV) Coronavirus .American journal of respiratory and critical care medicine 2020,201 (4):P7-P8.

3. Chan JF, Yuan S, Kok KH, To KK, Chu H, Yang J et al : A familial cluster of pneumonia associated with the 2019 novel coronavirus indicating person-to-person transmission: a study of a family cluster . Lancet 2020,395 (10223):514-523. 
4. Wu JT, Leung K, Leung GM:Nowcasting and forecasting the potential domestic and international spread of the 2019-nCoV outbreak originating in Wuhan, China: a modelling study . Lancet 2020,395 (10225):689-697.

5. Tian S, Hu N, Lou J, Chen K, Kang X, Xiang Z et al : Characteristics of COVID-19 infection in Beijing . The Journal of infection 2020,80 (4):401-406.

6. Xu L, Liu J, Lu M, Yang D, Zheng X:Liver injury during highly pathogenic human coronavirus infections . Liver international : official journal of the International Association for the Study of the Liver 2020 .

7. Zhou F, Yu T, Du R, Fan G, Liu Y, Liu Z et al : Clinical course and risk factors for mortality of adult inpatients with COVID-19 in Wuhan, China: a retrospective cohort study . Lancet 2020,395 (10229):1054-1062.

8. Parikh P, Patel J, Ingle M, Sawant P: Serum ferritin levels predict histological severity in patients with nonalcoholic fatty liver disease in India. Indian journal of gastroenterology : official journal of the Indian Society of Gastroenterology 2015, 34 (3):200-208.

9. Hamed AA, Darwish YW, El-Sayed MH:ADAMTS13 Levels in Young Patients With betaThalassemia Major: Relation to Hepatitis C Virus Infection, Liver Cirrhosis, and Iron Overload . Clinical and applied thrombosis/hemostasis : official journal of the International Academy of Clinical and Applied Thrombosis/Hemostasis 2015, 21 (6):527-532.

10. Wu SJ, Zhang ZZ, Cheng NS, Xiong XZ, Yang L: Preoperative serum ferritin is an independent prognostic factor for liver cancer after hepatectomy . Surgical oncology 2019, 29 :159-167.

11. Huang C, Wang Y, Li X, Ren L, Zhao J, Hu Y et al : Clinical features of patients infected with 2019 novel coronavirus in Wuhan, China . Lancet2020, 395 (10223):497-506.

12. Chen N, Zhou M, Dong X, Qu J, Gong F, Han Y et al : Epidemiological and clinical characteristics of 99 cases of 2019 novel coronavirus pneumonia in Wuhan, China: a descriptive study . Lancet 2020,395 (10223):507-513.

13. Yao XH, Li TY, He ZC, Ping YF, Liu HW, Yu SC et al : [A pathological report of three COVID-19 cases by minimally invasive autopsies]. Zhonghua bing li xue za zhi = Chinese journal of pathology 2020, 49 (0):E009.

14. Liu Z, Xiao X, Wei X, Li J, Yang J, Tan H et al: Composition and divergence of coronavirus spike proteins and host ACE2 receptors predict potential intermediate hosts of SARS-CoV-2 . Journal of medical virology 2020.

15. Walter-Nuno AB, Taracena ML, Mesquita RD, Oliveira PL, Paiva-Silva GO: Silencing of Iron and Heme-Related Genes Revealed a Paramount Role of Iron in the Physiology of the Hematophagous Vector Rhodnius prolixus . Frontiers in genetics 2018, 9 :19.

16. Brito-Azevedo A: High ferritin concentration in acute liver failure: The top of the iceberg?Liver international : official journal of the International Association for the Study of the Liver 2018, 38 (1):190.

Tables

Table 1 Univariate analysis of clinical characteristics of mild COVID-19 patients and recovery positive Patients

\begin{tabular}{llll}
\hline COVID-19 Patients & $\begin{array}{l}\text { Digestive tract symptoms } \\
\text { Positive cases/Total }\end{array}$ & $\begin{array}{l}\text { Respiratory symptoms } \\
\text { Positive cases/Total }\end{array}$ & $\begin{array}{l}\text { Fever(>37) Positive } \\
\text { cases/Total }\end{array}$ \\
\hline
\end{tabular}

Primary 


\begin{tabular}{llll}
\hline COVID-19 Patients & $\begin{array}{l}\text { Digestive tract symptoms } \\
\text { Positive cases/Total }\end{array}$ & $\begin{array}{l}\text { Respiratory symptoms } \\
\text { Positive cases/Total }\end{array}$ & $\begin{array}{l}\text { Fever( }>37) \text { Positive } \\
\text { cases/Total }\end{array}$ \\
\hline Cases (n) & $58 / 149$ & $118 / 149$ & $123 / 149$ \\
Rate (\%) & 38.93 & 79.19 & 82.55 \\
Recovery positive & & & \\
Cases (n) & $7 / 35$ & $13 / 35$ & $8 / 35$ \\
Rate (\%) & 20 & 48.57 & 22.86 \\
P value & 0.035 & $<0.001$ & $<0.001$ \\
\hline
\end{tabular}

Digestive tract symptoms: Nausea, vomiting, diarrhea, abdominal pain and anorexia

Respiratory symptoms: Cough, sputum, difficulty breathing, chest tightness, throat discomfort

Table 2 . Univariate analysis of serological indicators of mild COVID-19 patients and recovery positive Patients

\begin{tabular}{llll}
\hline Variables & Mild COVID-19 Patients & Recovery positive Patients & P \\
\hline Number & 149 & 35 & \\
Age (years) & $51.12 \pm 16.23$ & $45.69 \pm 14.54$ & 0.18 \\
Gender (F/M) & $78 / 71$ & $22 / 13$ & 0.26 \\
PT & $13.78 \pm 2.13$ & $12.22 \pm 0.90$ & 0.001 \\
Fibrinogen & $2.58 \pm 0.77$ & $2.24 \pm 0.59$ & 0.01 \\
APTT & $32.11 \pm 6.06$ & $29.98 \pm 4.99$ & 0.011 \\
TT & $13.57 \pm 2.07$ & $13.20 \pm 1.05$ & 0.08 \\
WBC & $5.37 \pm 1.81$ & $5.28 \pm 1.23$ & 0.73 \\
RBC & $4.02 \pm 0.59$ & $4.41 \pm 0.74$ & $<0.001$ \\
HGB & $119.60 \pm 18.93$ & $127.8 \pm 15.03$ & 0.001 \\
PLT & $223.45 \pm 79.06$ & $234.72 \pm 71.96$ & 0.28 \\
NEUT & $3.48 \pm 1.85$ & $3.12 \pm 0.90$ & 0.052 \\
LYMPH & $1.49 \pm 0.61$ & $1.59 \pm 0.59$ & 0.29 \\
MONO & $0.44 \pm 0.25$ & $0.44 \pm 0.12$ & 0.85 \\
EO & $0.079 \pm 0.086$ & $0.15 \pm 0.20$ & 0.008 \\
TCa & $2.15 \pm 0.23$ & $2.26 \pm 0.11$ & $<0.001$ \\
FE & $10.07 \pm 6.53$ & $17.03 \pm 6.62$ & $<0.001$ \\
ALT & $25.90 \pm 25.94$ & $21.50 \pm 16.13$ & 0.19 \\
AST & $25.43 \pm 18.01$ & $20.99 \pm 11.27$ & 0.06 \\
ALP & $70.10 \pm 28.06$ & $71.37 \pm 33.04$ & 0.77 \\
GGT & $31.44 \pm 25.34$ & $27.57 \pm 16.12$ & 0.24 \\
TP & $69.32 \pm 5.79$ & $72.47 \pm 5.78$ & 0.001 \\
ALB & $39.27 \pm 4.79$ & $41.03 \pm 3.63$ & 0.007 \\
GLO & $34.85 \pm 34.52$ & $31.44 \pm 4.05$ & 0.41 \\
A/G & $1.53 \pm 1.74$ & $1.32 \pm 0.18$ & 0.33 \\
PA & $275.68 \pm 88.94$ & $344.71 \pm 50.43$ & 0.001 \\
TBIL & $8.93 \pm 4.54$ & $8.73 \pm 2.87$ & 0.74 \\
DBIL & $2.46 \pm 2.87$ & $2.15 \pm 1.13$ & 0.39 \\
TBA & $6.50 \pm 2.64$ & $6.58 \pm 2.48$ & 0.82 \\
5'-nucleotide & $5.17 \pm 6.01$ & $4.15 \pm 2.08$ & 0.17 \\
BUN & $4.26 \pm 1.79$ & $4.49 \pm 2.82$ & 0.55 \\
CRE & $62.99 \pm 31.12$ & $53.19 \pm 17.62$ & 0.015 \\
UA & $279.26 \pm 84.30$ & $309.23 \pm 71.74$ & $<011$ \\
FE*PA & $2838.46 \pm 2255.32$ & $5969.25 \pm 2546$ & $<0.001$ \\
\hline & & &
\end{tabular}


NE, Neutrophils; TP, Total protein; ALB, Albumin; GLO, Globulin; PA, Pre-albumin; CRE, Creatinine; UA, uric acid; LYMPH, Lymphocyte; FE, Ferritin; DBIL, Direct bilirubin.

Table 3 . Correlation coefficients for the relationships between FE and various parameters of mild COVID-19 patients and recovery positive Patients

\begin{tabular}{lll}
\hline Factors & $\mathbf{R}$ & $P$ value \\
\hline PT & -0.26 & $<0.001$ \\
Fibrinogen & -0.24 & 0.001 \\
APTT & -0.34 & $<0.001$ \\
RBC & 0.33 & $<0.001$ \\
HGB & 0.40 & $<0.001$ \\
LYMPH & 0.27 & $<0.001$ \\
EO & 0.19 & 0.008 \\
TCa & 0.35 & $<0.001$ \\
TP & 0.18 & 0.017 \\
ALB & 0.16 & 0.031 \\
PA & 0.5 & $<0.001$ \\
TBIL & 0.15 & 0.042 \\
TBA & 0.31 & $<0.001$ \\
UA & 0.38 & $<0.001$ \\
\hline
\end{tabular}

Table 4. Multivariate analysis of factors associated with mild COVID-19 patients and recovery positive Patients

\begin{tabular}{llll}
\hline Factors & Odds ratio & $\mathbf{9 5 \%}$ CI & $P$ value \\
\hline PT & 0.55 & $0.36-0.85$ & 0.007 \\
FE & 1.22 & $1.12-1.32$ & $<0.001$ \\
ALT & 0.97 & $0.94-1.00$ & 0.026 \\
TBIL & 0.79 & $0.64-0.98$ & 0.031 \\
LYMPH & 0.32 & $0.12-0.84$ & 0.020 \\
DBIL & 1.76 & $1.15-2.69$ & 0.009 \\
FE*PA & 1.00 & $1.00-1.01$ & $<0.001$ \\
\hline
\end{tabular}

Figure Legends

Figure 1 Scatter diagram of (A) FE, (B) PA, (C) FE*PA, (D) APTT, (E) ALB and (F) HGB distribution between the primary mild and recovered COVID-19 patients.

Figure 2 Predictive nomogram for differentiation between primary mild and recovery positive COVID-19 patients.

Figure 3 The value of FE*PA levels was effective to discriminate between primary mild and recovery positive cases. A, The analysis of receiver operating characteristics (ROC) curve for FE. B, The analysis of ROC curve for FE. C. The analysis of ROC curve for FE*PA. 

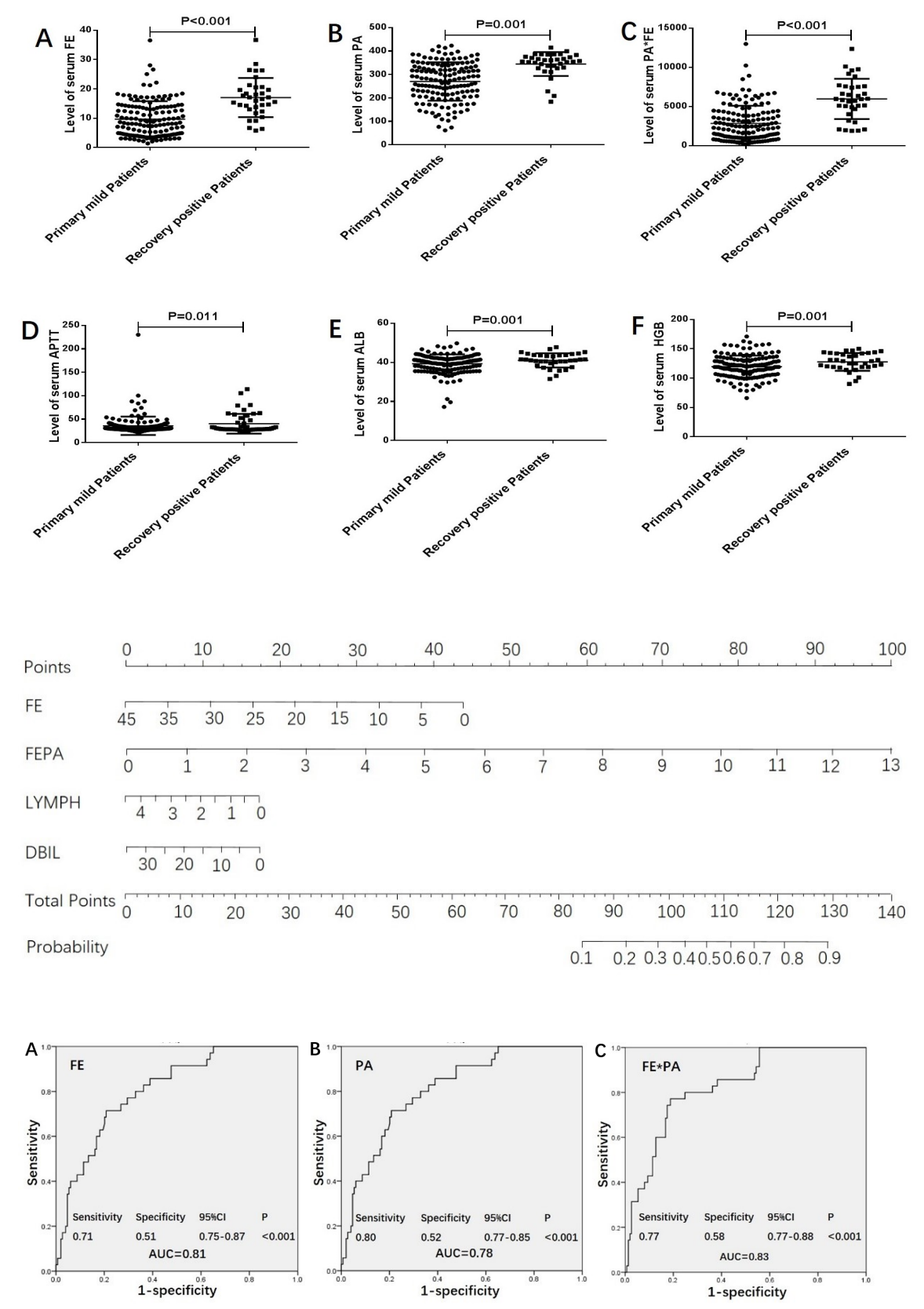